\title{
Point Prevalence Survey of Benzodiazepine and Sedative-Hypnotic Drug Use in Hospitalized Adult Patients
}

\author{
Heather L Neville, Mia Losier, Jennifer Pitman, Melissa Gehrig, Jennifer E Isenor, Laura V Minard, \\ Ellen Penny, and Susan K Bowles
}

\begin{abstract}
Background: Benzodiazepines and sedative-hypnotic drugs (BZD/ SHDs), such as zopiclone and the antidepressant trazodone, pose risks such as falls, fractures, and confusion, especially for older adults. Use of these drugs in the acute care setting is poorly understood.

Objectives: To determine the point prevalence and characteristics of use of BZD/SHDs in hospitals in Nova Scotia, Canada.

Methods: A point prevalence survey was conducted for adults admitted to all hospitals with at least 30 acute care beds between May and August 2016. Drugs administered intravenously, patients in long-term care, and patients receiving mental health services, addiction treatment, or critical care were excluded. The proportion of included patients who had received a BZD/SHD within the $24 \mathrm{~h}$ before the start of the survey was determined. A descriptive statistical analysis was performed.
\end{abstract}

Results: Overall BZD/SHD prevalence was 34.6\% (487/1409) across the 16 eligible hospitals. The average age was 70.3 years, and $150(30.8 \%)$ of the patients were 80 years or older. Among the 585 prescriptions for these patients, commonly used drugs were zopiclone (32.0\%), lorazepam (21.9\%), and trazodone (21.9\%). The most common indications for use were bedtime/daytime sedation (60.0\%) and anxiety (12.5\%). More than half of the prescriptions (55.7\%) had been initiated at home, $37.6 \%$ were started in hospital, and the place of initiation was unknown for $6.7 \%$. Benzodiazepines were prescribed more frequently to patients under 65 years than those 80 years or older $(41.3 \%$ versus $22.2 \%, p<0.001)$ whereas trazodone was more frequently prescribed to the older of these 2 age groups ( $52.7 \%$ versus $14.3 \%, p<0.001)$.

Conclusions: BZD/SHDs were frequently used by hospitalized adult patients in Nova Scotia. Trazodone appears to have been substituted for benzodiazepines in the oldest age group. Pharmacists should direct their efforts toward preventing inappropriate initiation of BZD/SHDs in hospital, particularly for elderly patients.

Keywords: benzodiazepines, sedative-hypnotic drugs, trazodone, hospital, geriatrics, sleep

\section{RÉSUMÉ}

Contexte : Les benzodiazépines et les médicaments sédatifs-hypnotiques (BZD/MSH), comme la zopiclone et l'antidépresseur trazodone, comportent des risques de chute, de fracture et de confusion, particulièrement chez les personnes âgées. Il existe une mauvaise compréhension de l'utilisation de ces médicaments dans un contexte de soins intensifs.

Objectifs : Déterminer la prévalence ponctuelle et les caractéristiques de l'utilisation des BZD/MSH dans des hôpitaux en Nouvelle-Écosse, au Canada.

Méthodes : Une enquête sur la prévalence ponctuelle a été menée entre mai et août 2016 auprès d'adultes admis dans les hôpitaux comptant au moins 30 lits en soins intensifs. Les patients recevant ces médicaments par voie intraveineuse, ceux en établissement de soins de longue durée, ceux recevant des services en santé mentale ou un traitement pour la toxicomanie ou encore ceux des soins intensifs ont été exclus de l'enquête. La détermination de la proportion des patients inclus dans l'étude portait sur ceux qui avaient reçu des BZD/MSH au cours des 24 h précédant le début de l'enquête, et elle a été suivie d'une analyse statistique descriptive.

Résultats : De manière générale, l'usage des BZD/MSH s'élevait à 34,6 \% (487/1409) dans les 16 hôpitaux participants. L'âge moyen des patients était de 70,3 ans et 150 (30,8\%) étaient âgés d'au moins 80 ans. Parmi les 585 prescriptions pour ces patients, les médicaments communément utilisés étaient la zopiclone $(32,0 \%)$, le lorazepam $(21,9 \%)$ et le trazodone $(21,9 \%)$. Les indications d'utilisation les plus répandues concernaient la sédation au coucher et en cours de journée $(60 \%)$ et l'anxiété (12,5\%). Plus de la moitié des prescriptions (55,7\%) ont commencé à domicile, 37,6 \% ont commencé à l'hôpital, et le lieu du début de la prise de ces médicaments était inconnu dans $6,7 \%$ des cas. La prescription des benzodiazépines s'adressait plus souvent aux patients de moins de 65 ans qu'à ceux d'au moins 80 ans (41,3\% par rapport à $22,2 \%, p<0,001)$, tandis que la prescription de trazodone s'adressait plus souvent aux personnes de la tranche d'âge plus avancée $(52,7 \%$ par rapport à $14,3 \%, p<0,001)$. 


\section{Can J Hosp Pharm. 2020;73(3):193-201}

Conclusions : Les BZD/MSH étaient fréquemment utilisés par les patients adultes hospitalisés en Nouvelle-Écosse. La trazodone semble avoir remplacé les benzodiazépines dans le groupe plus âgé. Les pharmaciens devraient orienter leurs efforts sur la prévention de la prise inappropriée des BZD/MSH en hôpital, particulièrement par les patients plus âgés.

Mots-clés : benzodiazépines, médicaments sédatifs-hypnotiques, trazodone, hôpital, gériatrie, sommeil

\section{INTRODUCTION}

Cleep disturbances and anxiety are common in acutely ill, Ohospitalized adults. ${ }^{1,2}$ The hospital environment and clinical care, such as blood tests and vital sign checks, can combine with the patients' illness, pain, reduced mobility, and medication adverse effects to disturb sleep. ${ }^{3}$ Benzodiazepines (BZDs) and other sedative-hypnotic drugs (SHDs), specifically benzodiazepine receptor agonists (also called z-drugs), are commonly used to treat anxiety and insomnia. Trazodone, an antidepressant, is also frequently prescribed to adults for sleep. ${ }^{4,5}$ However, use of these drug classes poses concerns in the acute care setting, including increased risk of falls, fractures, and hospital readmission, especially for older adults. ${ }^{6-9}$ In some studies, hospitalized patients taking BZDs and z-drugs had higher odds of falling and experiencing severe injury. ${ }^{7,10}$ At a rural hospital in Alberta, Canada, $55 \%$ of patients who had experienced a fall in hospital had a prescription for a BZD, and 38\% had a prescription for an SHD such as zopiclone (a z-drug). ${ }^{11}$ Trazodone has also been associated with daytime sedation, orthostatic hypotension, priapism, and falls leading to emergency department visits or hospital admissions, even at the low doses typically used for sleep disturbances. ${ }^{5,12-14}$ As well, initiation of a BZD/SHD in the hospital setting can lead to continued use after discharge. ${ }^{15-18}$

Despite increased awareness of risks, deprescribing initiatives, and published guidelines that recommend avoiding the use of BZD/SHD in elderly patients, ${ }^{19-21}$ there has been no marked decrease in prescribing rates in the community. In Canada, the United Kingdom, and Europe, slight decreases in benzodiazepine use have often been offset by increases in the use of z-drugs and, less frequently, trazodone. ${ }^{14,22-26}$ In the United States, ambulatory visits associated with the prescribing of both benzodiazepines and z-drugs have increased among patients 65 years of age and older. ${ }^{27}$ Utilization of BZD/SHDs in hospital settings is usually more frequent than in community settings, with rates as high as $62 \% .^{15,28-31}$ It is not clear whether BZD rates are decreasing and/or BZDs are being substituted with other sedatives such as $\mathrm{z}$-drugs and trazodone in hospitals. Published studies of sedative prescribing patterns have been limited to a single drug class (for example, benzodiazepines), to individual wards, or to academic hospitals in urban settings, which do not provide a broad view of current utilization. ${ }^{15,16,28-30}$
To better understand the use of sedatives in the hospital setting, we sought to determine the utilization of benzodiazepines, z-drugs, and trazodone (collectively termed BZD/SHDs) in adult hospitals in Nova Scotia, Canada. The information gained will assist our hospital, and potentially other health care organizations, to identify targets for quality improvement, as well as providing a baseline measure against which to evaluate the effectiveness of future interventions. ${ }^{32,33}$ The primary objective of this study was to conduct a point prevalence survey of BZD/SHD utilization in the study hospitals. The secondary objectives were to determine the rate of initiation of BZD/SHDs in hospital and to characterize utilization in terms of specific drugs used, patients' age and sex, admitting service, hospital type, indication, dose, and whether the $\mathrm{BZD} / \mathrm{SHD}$ was ordered with a preprinted order (PPO).

\section{METHODS}

\section{Setting}

A cross-sectional point prevalence survey was conducted in Nova Scotia, a Canadian province with a population of $953900 .^{34}$ Canada has a universal public health care system in which medically essential services are provided by physicians and hospitals at no cost to patients. ${ }^{35}$ The Nova Scotia Health Authority (NSHA) delivers health care services to Nova Scotians and specialized adult care to other nearby provinces. ${ }^{36}$ The NSHA has one adult tertiary care centre, 9 regional hospitals, and more than 135 other community locations. ${ }^{37}$ In 2016, there were almost 3200 hospital beds and more than 1 million inpatient days. ${ }^{37}$ At the time of the survey, NSHA hospitals did not have a common drug formulary, electronic health record, or computerized physician order entry. Ethics approval to conduct the study was received on June 8, 2016, from the NSHA Research Ethics Board (file 1021365). Informed consent was not required because of the study design.

\section{Hospital and Patient Selection}

Hospitals with at least 30 acute care beds were included. Patients in transitional care (or alternate level of care) beds were included, because these patients are at risk due to frailty and older age. Transitional care patients are those who no longer require acute care services and are waiting for discharge to another setting, such as long-term care. ${ }^{38}$ Hospitals were categorized by location, 
according to the size of the population centre, as small and medium ( $<100000$ residents) or large ( $\geq 100000$ residents) ${ }^{39}$ Data were collected between June and August 2016. The survey of each individual ward was completed in 1 day, and surveys for all wards in a given hospital were completed within a 2-week period. Patient rosters were obtained from each hospital to identify the patients present on each audit day. Eligible patients were those 18 years of age or older who had been admitted for at least $24 \mathrm{~h}$ as of 0800 on the day of the survey. Patients were excluded if they had been admitted for psychiatric care, addiction treatment, or critical care, because these services would be associated with appropriate indications for sedatives (e.g., alcohol withdrawal, seizures, or conscious sedation). Long-term care residents and patients in the emergency department, whether visiting or waiting for admission to hospital, were also excluded.

Patients with documented administration of a benzodiazepine, a z-drug (zopiclone or zolpidem), or trazodone by the oral (including enteral or gastric tube), sublingual, subcutaneous, or intramuscular route, in the $24 \mathrm{~h}$ before 0800 on the day of the survey, were used to calculate the numerator. For the purposes of this study, medication use had to be documented on the patientspecific medication administration record. Other psychotropic drugs used off-label for sedation (e.g., antipsychotics) were not included because of the difficulty in determining the indication. Medications administered by the IV route were excluded because these would likely be associated with appropriate indications that were not of interest in this study (e.g., palliative care, critical care, alcohol withdrawal, treatment of seizures, or procedural use). The denominator was the total number of patients on the ward. Prevalence was calculated by dividing the numerator by the denominator for each population unit (e.g., ward, hospital, and province).

\section{Training and Data Collection}

A training manual was developed, and all co-investigators and 3 research assistants were trained in data collection procedures by the principal investigator (H.L.N.). The first 25 charts audited by each research assistant were assessed by the principal investigator for accuracy, and at least $10 \%$ of audited charts were assessed by a second member of the research team during the data collection period for quality control.

For patients with administration of a BZD/SHD according to the inclusion criteria, the following data were collected: age, sex, hospital admitting service, drug name, indication, dose, interval, route of administration, whether the medication was ordered on a PPO, total dose administered in the previous $24 \mathrm{~h}$, and in-hospital initiation of the drug. A PPO is a hospitalapproved set of orders designed to promote best practice and consistency in case management. For patients without administration of a BZD/SHD, no data were collected. The following ward information was collected: hospital name, ward name, specialty (e.g., medical), and number of beds. Orders for oxazepam, temazepam, trazodone, and z-drugs that were administered at bedtime were assumed to be given for sedation, because of the timing of the dose and the typical indications for these drugs. Data sources included the best possible medication history on admission, medication orders, records from the history and physical examination, progress notes, nursing notes, and medication administration records. All patients with administration of a BZD/SHD were given a unique study number; no personal identifiers were collected during the study.

\section{Statistical Analysis}

Data were entered into an Excel database (Microsoft Corporation, Redmond, Washington) by a research assistant and double-checked by a second research assistant. Results were

\section{Table 1. Patient Characteristics and Point Prevalence of Benzodiazepines and Sedative-Hypnotic Drugs (BZD/SHDs) at Acute Care Hospitals in Nova Scotia $(n=1409)$}

\begin{tabular}{|c|c|c|c|}
\hline \multirow[b]{2}{*}{ Characteristic } & \multicolumn{2}{|c|}{ Study Group; No. (\%) of Patients } & \multirow[b]{2}{*}{ Prevalence, \% } \\
\hline & $\begin{array}{c}\text { Received } \\
\text { a BZD/SHD }\end{array}$ & $\begin{array}{c}\text { Did Not Receive } \\
\text { a BZD/SHD }\end{array}$ & \\
\hline All patients & 487 & 922 & 34.6 \\
\hline Age (years) & & Data not collected & Data not collected \\
\hline$<65$ & $152(31.2)$ & & \\
\hline $65-79$ & $185(38.0)$ & & \\
\hline$>79$ & $150(30.8)$ & & \\
\hline Sex, female & $266(54.6)$ & Data not collected & Data not collected \\
\hline \multicolumn{4}{|l|}{ Population centre* } \\
\hline Large & $185(38.0)$ & $415(45.0)$ & 30.8 \\
\hline Small or medium & $302(62.0)$ & $507 \quad(55.0)$ & 37.3 \\
\hline \multicolumn{4}{|l|}{ Specialty } \\
\hline Medicine & $303(62.2)$ & $541(58.7)$ & 35.9 \\
\hline Surgery & $92(18.9)$ & $218(23.6)$ & 29.7 \\
\hline Transitional care & $41 \quad(8.4)$ & $70 \quad(7.6)$ & 36.9 \\
\hline Othert & $51(10.5)$ & 93 (10.1) & 35.4 \\
\hline
\end{tabular}


summarized descriptively, and categorical data were analyzed by $\chi^{2}$ tests with continuity correction for $2 \times 2$ tables to compare characteristics between groups of BZD/SHD users.

\section{RESULTS}

All 16 eligible hospitals participated in the point prevalence survey. Overall BZD/SHD point prevalence was $34.6 \%$

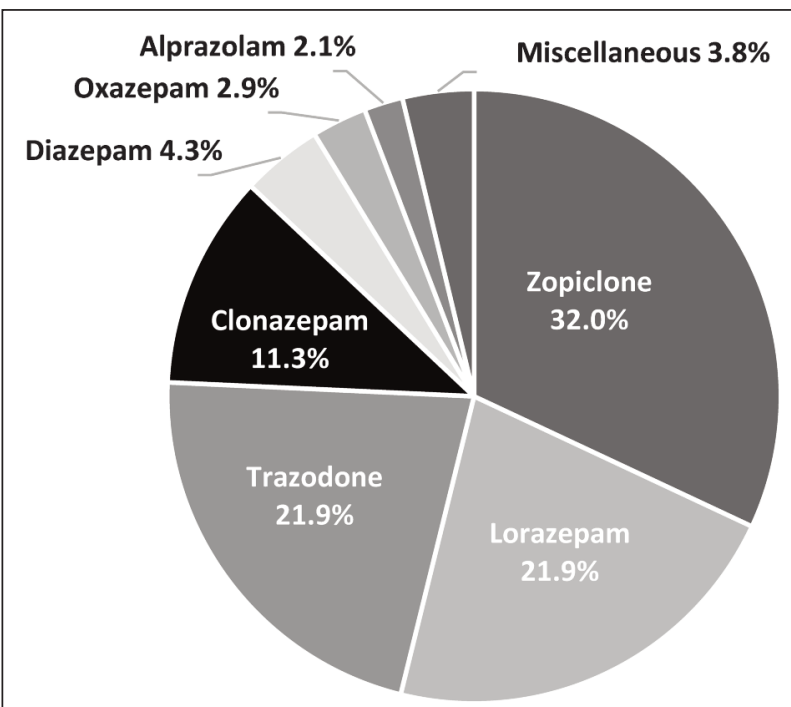

Figure 1. Specific benzodiazepine and sedativehypnotic drugs prescribed in Nova Scotia hospitals, as proportions of all prescriptions for this group of drugs $(n=585)$.

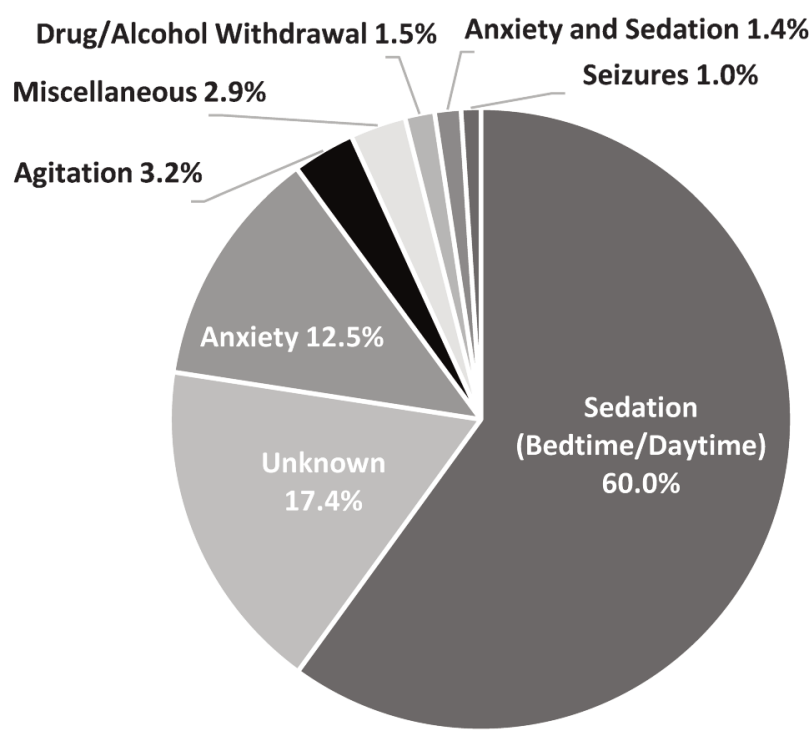

Figure 2. Indications for benzodiazepine and sedative-hypnotic drug prescriptions in Nova Scotia hospitals ( $n=585$ prescriptions).
(487/1409). The point prevalence was 37.3\% (302/809) for hospitals located in small and medium population centres, compared with 30.8\% (185/600) for hospitals in large population centres (Table 1). Prevalence was higher among patients in transitional care (36.9\% [41/111]) and medicine patients (35.9\% [303/844]) than among surgical patients (29.7\% [92/310]). Among the 487 patients who received a BZD/SHD, the mean age was 70.3 years (range 18-103 years), with most being over the age of $65(n=335$ [68.8\%]). The most common admitting service was medicine $(n=303$ [62.2\%]). Data collection by research assistants was audited by the research team for 97 (19.9\%) of included patients, and any discrepancies were resolved at the time of the audit.

There were a total of $585 \mathrm{BZD} / \mathrm{SHD}$ orders for the 487 patients, with 92 patients (18.9\%) receiving more than 1 agent. The most commonly administered drugs are shown in Figure 1. Benzodiazepines as a class were the most frequently used $\mathrm{BZD} / \mathrm{SHD}(n=270$ [46.2\%]), but the top individual agents were zopiclone $(n=187$ [32.0\%]), trazodone $(n=128$ [21.9\%]) and lorazepam $(n=128$ [21.9\%]). Indications for use are depicted in Figure 2. Administration for sedation ( $n=351$ prescriptions) occurred at bedtime ( $n=336$ [95.7\%]), both at bedtime and in the daytime $(n=13[3.7 \%])$, or in the daytime only $(n=2[0.6 \%])$.

Most BZD/SHDs were documented in written prescriptions $(n=549$ [93.8\%]), with relatively few documented in PPOs $(n=32[5.5 \%])$ or having unknown documentation $(n=4$ $[0.7 \%])$. Four prescriptions could not be found in the paper charts because earlier chart information could not be located for long hospital admissions. The PPOs listed BZD/SHDs for the following indications: sedation (23), alcohol withdrawal (6), anxiety (2), and procedural use (1). Of the 10 PPOs that listed zopiclone, 3 did not adjust the dose according to the patient's age. PPOs were most often used on medicine units (23/32 [71.9\%]).

Characteristics associated with whether the medications were initiated at home $(n=326[55.7 \%])$ or in the hospital $(n=220$ [37.6\%]) are presented in Table 2; for the remaining 39 orders (6.7\%), the initiation setting could not be determined. Among $\mathrm{BZD} / \mathrm{SHD}$ users, female sex and scheduled drug regimens were more frequently associated with the drugs being prescribed before admission ( $p=0.005$ and $p<0.001$, respectively). "Take when needed" (PRN) prescriptions and PPOs were more frequently associated with the drugs being prescribed in hospital $(p<0.001$ for both).

Patients' age distribution and BZD/SHD dosing information are provided in Table 3 and Table 4, respectively. Benzodiazepines were more frequently prescribed to patients less than 65 years old compared with patients 80 years of age or older $(41.3 \%$ versus $22.2 \%, p<0.001$ ), whereas trazodone was more frequently prescribed to patients 80 years of age or older compared with patients less than 65 years old $(52.7 \%$ versus $14.3 \%, p<0.001)$. Prescription 
Table 2. Prescriptions for Benzodiazepine and Sedative-Hypnotic Drugs in Relation to Setting Where Drug Was Initiated $(n=546)$

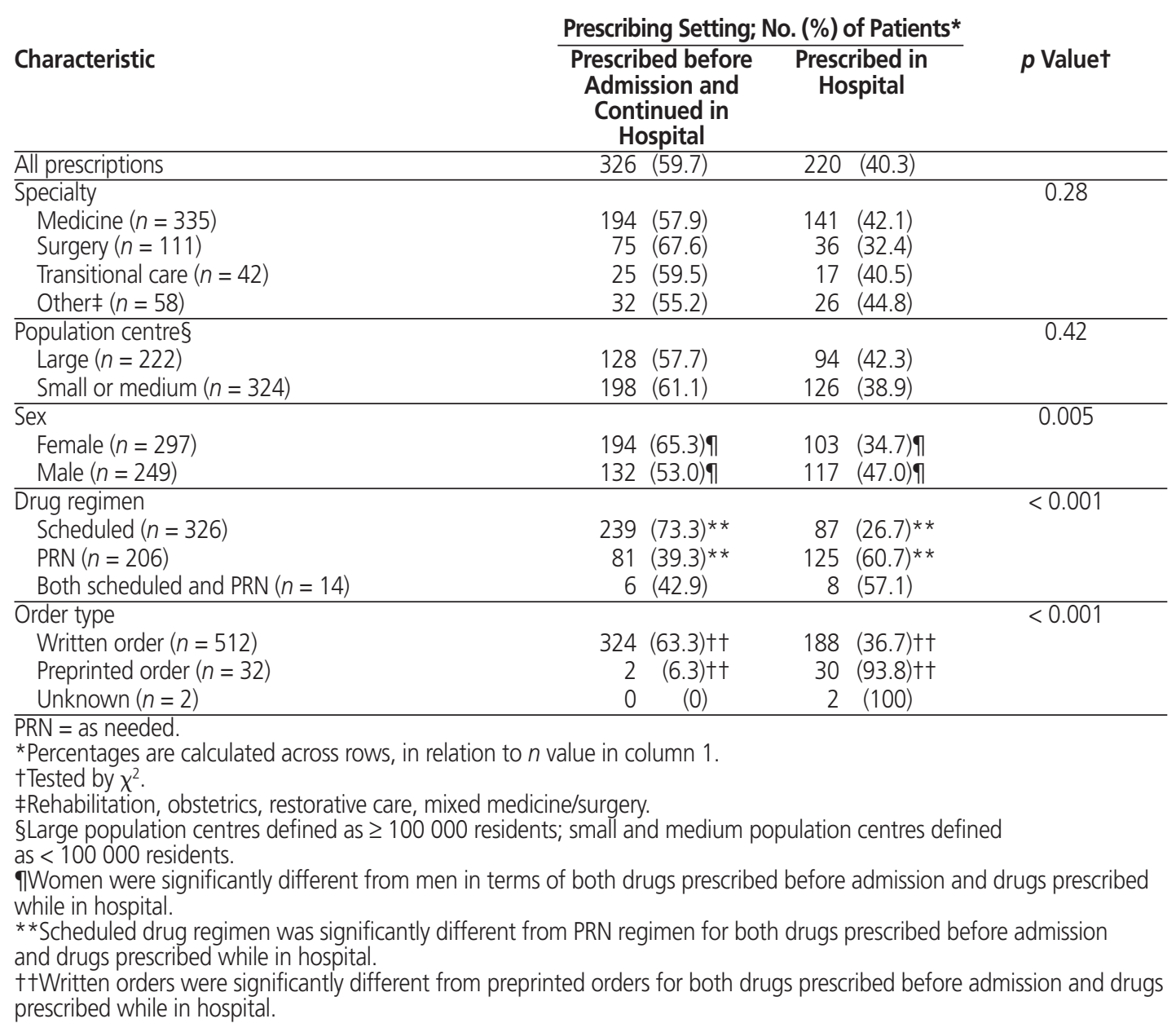

Table 3. Benzodiazepine and Sedative-Hypnotic Drugs Prescribed by Age Category in Nova Scotia Hospitals

\begin{tabular}{|c|c|c|c|c|c|}
\hline \multirow[b]{2}{*}{ Drugs Prescribed* } & \multicolumn{4}{|c|}{ Age Group; No. (\%) of Patientst } & \multirow[b]{2}{*}{$p$ Value‡ } \\
\hline & $<65$ years & $65-79$ years & $\geq 80$ years & All Patients & \\
\hline All patients & 152 & 185 & 150 & 487 & $<0.001$ \\
\hline Benzodiazepine only & 69 (41.3)§ & $61 \quad(36.5)$ & $37(22.2) \S$ & 167 & \\
\hline Trazodone only & 13 (14.3)ף & $30 \quad(33.0)$ & $48(52.7) \uparrow$ & 91 & \\
\hline Zopiclone only & $37(27.0)$ & $52 \quad(38.0)$ & $48(35.0)$ & 137 & \\
\hline Any 2 sedatives & $31(36.0)^{* *}$ & $38(44.2)^{* *}$ & $17(19.8)^{\star *}$ & 86 & \\
\hline Any 3 sedatives & $2(33.3)$ & $4 \quad(66.7)$ & 0 & 6 & \\
\hline
\end{tabular}

*Patients were divided into 5 mutually exclusive groups: benzodiazepine (any), trazodone, zopiclone, any 2 sedatives (any combination, including multiple benzodiazepines), and any 3 sedatives (any combination, including multiple benzodiazepines).

tPercentages are calculated across rows, in relation to total number of patients in each mutually exclusive group based on drugs prescribed.

¥Tested by $\chi^{2}$.

§Prescription of benzodiazepines was significantly different for age group $<65$ years compared with age group $\geq 80$ years.

IPrescription of trazodone was significantly different for age group $\geq 80$ years compared with age group $<65$ years.

**Prescription of 2 sedatives was significantly different for age groups $<65$ years and $65-79$ years compared with age group $\geq 80$ years.

of 2 sedatives at the same time was significantly associated with patients less than 65 years old and 65-79 years old compared with patients 80 years of age or older. As well, the mean dose decreased with increasing age, except for trazodone, for which the mean dose was $52.73 \mathrm{mg}$ in patients 80 years of age or older and $51.68 \mathrm{mg}$ in patients 65 to 79 years of age.

\section{DISCUSSION}

This point prevalence survey showed that $\mathrm{BZD} / \mathrm{SHD}$ s were administered to approximately $35 \%$ of hospitalized patients in Nova Scotia over a $24-\mathrm{h}$ period. This represents a substantial proportion of the general population in hospitals, given the 
This single copy is for your personal, non-commercial use only.

For permission to reprint multiple copies or to order presentation-ready copies for distribution, contact CJHP at publications@cshp.ca

Table 4. Mean Dose* by Age Category for Benzodiazepine and Sedative-Hypnotic Drugs in Nova Scotia Hospitals

\begin{tabular}{|c|c|c|c|c|c|c|}
\hline \multirow[b]{2}{*}{ Drugt } & \multicolumn{2}{|c|}{ Age $<65$ years } & \multicolumn{2}{|c|}{ Age $65-79$ years } & \multicolumn{2}{|r|}{ Age $\geq 80$ years } \\
\hline & $n$ & Mean Dose \pm SD & $n$ & Mean Dose \pm SD & $n$ & Mean Dose \pm SD \\
\hline Clonazepam & 23 & $1.07 \pm 0.87$ & 36 & $0.61 \pm 0.43$ & 7 & $0.50 \pm 0.25$ \\
\hline Lorazepam & 62 & $1.12 \pm 0.47$ & 36 & $1.04 \pm 0.48$ & 30 & $0.89 \pm 0.39$ \\
\hline Trazodone & 21 & $84.52 \pm 51.52$ & 52 & $51.68 \pm 33.58$ & 55 & $52.73 \pm 28.33$ \\
\hline Zopiclone & 57 & $7.54 \pm 2.97$ & 72 & $6.65 \pm 2.04$ & 58 & $6.44 \pm 2.52$ \\
\hline
\end{tabular}

$\mathrm{SD}=$ standard deviation.

* Mean total dose $(\mathrm{mg})$ administered in the previous $24 \mathrm{~h}$, or maximum prescribed dose if the dose was prescribed as a range and actual dose administered was not documented in the medication administration record (for which the following number of cases occurred: $z$ opiclone, $n=5$; trazodone, $n=7$; lorazepam, $n=3$ ).

tData for other drugs were suppressed because of small sample sizes $(\leq 5)$.

specific groups that were excluded from this analysis (e.g., critically ill patients, those receiving mental health services). The majority of use was for bedtime sedation of older patients on medicine units. Despite the well-known risks of BZD/SHDs, these results indicate that prevalence has not changed very much in the past 15 years, since the publication of 2 previous cross-sectional surveys in 2001 and $2005 .{ }^{40,41}$ Those earlier surveys reported that benzodiazepines were prescribed for $21.2 \%$ to $36 \%$ of patients in the hospital setting, although actual administration to patients was not described. ${ }^{40,41}$ In a more recent point prevalence survey involving an audit of various psychotropic medications, conducted in 2014, Brunero and others ${ }^{28}$ reported that benzodiazepines were prescribed for $10 \%$ of patients. Those authors found that indications were not documented in $60 \%$ of prescriptions, a rate much higher than what was observed in our study. As well, $56 \%$ of the prescriptions in the earlier study were started in the hospital, compared with $37.6 \%$ in our point prevalence survey. Brunero and others ${ }^{28}$ found that $44 \%$ of all psychotropics were continued upon discharge, whereas this type of information was not collected in our study.

Importantly, just over half of the BZD/SHDs prescribed to Nova Scotia inpatients were 2 non-benzodiazepine sedatives, zopiclone and trazodone, which supports community data that these drugs are replacing benzodiazepines. ${ }^{14,22-25}$ Antipsychotics such as quetiapine, although not included in this study, are also replacing benzodiazepines because of their sedative properties. ${ }^{14,16}$ Few reports have highlighted benzodiazepine substitution in hospitals. In a study conducted in 2013, Gillis and others ${ }^{16}$ found that trazodone was the most commonly administered sedative in a hospital in Boston, Massachusetts (30\% of patients), followed by lorazepam (24\%) and z-drugs (18\%). Although that study was a retrospective review of sedative use over a 2 -month period, and not a point prevalence survey, the hospital's electronic medication administration system (rather than patient records) was used to document actual administered doses. Only a small number of patients were excluded from the study because the medication was ordered but not administered (15 out of 276 screened). ${ }^{16}$ In contrast, Arnold and others ${ }^{30}$ reviewed patient records over a 3-month period for psychotropic drugs administered to patients and found prevalences of $0.2 \%$ for trazodone and $12.6 \%$ for z-drugs. As well, when Schumacher and others ${ }^{15}$ prospectively reviewed sedative drug use over a 3-month period using electronic medication administration data, they found low utilization of trazodone (1\%) and z-drugs (11\%).

It has been hypothesized that clinicians consider z-drugs and trazodone to be safer alternatives to benzodiazepines, particularly for elderly patients. ${ }^{5,31}$ When we analyzed our data by age, we found that patients 80 years of age or older were more likely to have a prescription for trazodone and less likely to have a prescription for benzodiazepines alone or 2 different sedatives. Zopiclone use was fairly evenly distributed among all age groups. In the community setting, Alessi-Severini and others ${ }^{22}$ found that $z$-drug use increased and BZD use decreased among residents over the age of 65 years over a 16 -year period. Iaboni and others ${ }^{14}$ compared changes in sedative use in community and long-term care settings from 2002 to 2013 in Ontario, Canada. They found that BZD use decreased while low-dose trazodone use increased among older adults. Overall, psychotropic drug use decreased among community-dwelling seniors, but those in long-term care received increasing numbers of prescriptions, due to significant increases in trazodone..$^{14}$ In our study, it was encouraging to note that a combination of 2 or more sedatives was prescribed less frequently to patients 80 years of age or older, in contrast to findings that psychotropic polypharmacy has increased among older adults in other settings. ${ }^{14}$

Elderly people are at greater risk of adverse effects from BZD/SHD use, particularly at higher doses. ${ }^{42}$ Published guidelines, such as the Beers Criteria ${ }^{20}$ and the Screening Tool of Older Person's Prescriptions (STOPP), ${ }^{21}$ recommend against the use of BZDs and z-drugs because of the risk of delirium, cognitive impairment, falls, fractures, and motor vehicle accidents. Trazodone is not specifically mentioned in these guidelines; however, it would be covered by the Beers Criteria recommendation to minimize the use of central nervous system-active drugs to reduce the risk of falls. ${ }^{20}$ The Choosing Wisely Canada toolkit for reducing BZD/SHD use by hospitalized older adults further reinforces the message that these medications are not the first choice for insomnia, agitation, or delirium. ${ }^{43}$ In acute care hospitals in Nova Scotia, almost $70 \%$ of patients who received a $\mathrm{BZD} / \mathrm{SHD}$ were over the age of 65 , and $30 \%$ were over the age 
of 80. Evaluation of average daily doses administered for the top 4 drugs in our study indicated that for most patients the dose did decrease with age; however, the mean zopiclone doses of $6.65 \mathrm{mg}$ for patients 65 to 79 years of age and $6.44 \mathrm{mg}$ for those 80 years or older (Table 4) were higher than recommended in the product monograph. ${ }^{44}$ Canadian dosing guidelines suggest that for patients over 65 years of age, zopiclone should be started at $3.75 \mathrm{mg}$ and titrated to $5 \mathrm{mg}$, to reduce the risk of impaired cognitive function and coordination, which have led to confusion and falls. ${ }^{44}$

Our point prevalence survey showed that BZD/SHDs were newly prescribed in hospital for $35 \%$ of patients, which is concerning because of the risk that sedatives initiated in hospital may be continued upon discharge. ${ }^{16-18,45}$ Initiation of therapy in hospital is likely multifactorial, but could be triggered by use of a PPO. In our survey, only 32 orders originated from PPOs, but $94 \%$ of them were for newly prescribed BZD/SHDs. PPOs were more likely to be used in medicine wards. Freter and others ${ }^{46}$ reported a PPO intervention to reduce delirium among orthopedic patients in one of the hospitals included in the current study. In the earlier study, intervention PPOs focused on scheduled analgesics and laxatives, trazodone (instead of benzodiazepines) for nighttime sedation, and other protocols. Benzodiazepine use was negligible, which the authors attributed to benzodiazepines not being listed on the PPOs and to previous educational efforts at the hospital. However, zopiclone (also not listed on the PPOs) was prescribed for patients in both intervention and control groups ${ }^{46} \mathrm{~A}$ retrospective chart review of sedative use by patients in another Canadian hospital found that $20.4 \%$ of potentially inappropriate prescriptions were ordered from a PPO set on admission or postoperatively. ${ }^{29}$ These results suggest a quality improvement opportunity to review the use of sedatives listed on PPOs.

For most of the patients in our study, sedatives had been prescribed before admission, and this therapy was continued while they were in hospital; this finding was even more likely for female patients. It can be challenging to address long-term home therapy in the acute care setting: the patients are sick, hospital stays are often short, and safe withdrawal of a BZD/SHD can require weeks to months of dose tapering. Despite these challenges, a hospital admission may present an opportunity to initiate discontinuation of long-term BZD/SHD use. In one recent Canadian pilot study involving 50 patients over the age of 65 years, $64 \%$ successfully discontinued sedative drugs by 30 days after discharge without an increase in sleep-related disturbances. ${ }^{47}$ Hospitalized patients who were occasional (3-6 times a week) and regular (daily) users were given educational material with instructions for tapering and stopping long-term sedative use. The deprescribing intervention was more successful for patients who started the tapering process in hospital $(29 / 36,81 \%)$ than for those who started after discharge $(3 / 14,21 \%) .{ }^{47}$ Hospital-based initiatives could be combined with a community-based public health approach to deprescribing BZD/SHDs, although currently there is uncertainty about how this can best be accomplished. ${ }^{31}$ Research does support a stepwise approach, such as engaging the patient and health care providers, initiating trials of dose tapering, employing nonpharmacologic treatment such as cognitive behavioural therapy, and seeking specialist help when indicated. ${ }^{19,31,43,48}$ Pharmacists can play a leading role in hospital-based interventions, particularly in terms of providing patient education, advising on safe tapering protocols, and communicating and following up with primary care providers.

Limitations to our point prevalence survey included reliance on documentation in the health record and potential inaccuracy of data collection. We sought to minimize errors by doublechecking at least $10 \%$ of charts and requiring independent data entry. Demographic characteristics (e.g., age, sex) of patients who did not receive BZD/SHDs were not collected, which restricted our analysis to those patients who used a BZD/SHD and thus limited our interpretation of the findings. As well, some drugs that might have been administered for sedation (e.g., antipsychotics, antihistamines, melatonin) were not audited. Antipsychotics are frequently prescribed to hospital patients for sedation but also for other indications. ${ }^{14,16}$ Data collection occurred during the summer months when bed occupancy may be lower; however, the sample was still expected to be representative. A point prevalence survey measures drug use at one point in time; therefore, we were not able to follow patients to discharge to determine whether BZD/SHDs were continued. Finally, we assumed that when oxazepam, z-drugs, temazepam, and trazodone were given at bedtime, they were given for sedation and not for other indications (e.g., anxiety or to manage behaviour).

The strengths of this study included the comprehensive sample and the large group of medications surveyed. All eligible hospitals in Nova Scotia participated, representing small, medium, and large population centres. The survey results are therefore generalizable to the entire province and likely to other jurisdictions. Selected populations were purposively excluded (e.g., patients receiving mental health services, addictions treatment, and critical care), because these represented settings where use of these drugs is often for other indications, such as seizures or alcohol withdrawal. This allowed us to focus on areas where BZD/SHD use for insomnia and anxiety has been more problematic (e.g., medical and surgical settings).

\section{CONCLUSION}

This point prevalence survey showed that $\mathrm{BZD} / \mathrm{SHD}$ s were frequently used by hospitalized patients in Nova Scotia, Canada, most of whom were 65 years of age or older. Two nonbenzodiazepine drugs, zopiclone and trazodone, were responsible for more than $50 \%$ of sedatives prescribed. Pharmacists and other health care providers should direct their efforts toward reducing 
$\mathrm{BZD} / \mathrm{SHD}$ use in the community, preventing inappropriate initiation of BZD/SHD in hospital, and, when these drugs are used, prescribing the lowest effective dose to minimize harm and improve quality of patient care. Future research should examine all drugs that might potentially be used for sleep, such as antipsychotics, antidepressants, antihistamines, and melatonin. To successfully implement interventions aimed at reducing BZD/ SHD use in hospital, it will be important to better understand the perspectives of patients, prescribers, and other health care providers and to identify facilitators of and barriers to use.

\section{References}

1. Young JS, Bourgeois JA, Hilty DM, Hardin KA. Sleep in hospitalized medical patients, part 1: factors affecting sleep. J Hosp Med. 2008;3(6):473-82.

2. AlBekairy A, AbuRuz S, Alsabani B, Alshehri A, Aldebasi T, Alkatheri A, et al. Exploring factors associated with depression and anxiety among hospitalized patients with type 2 diabetes mellitus. Med Princ Pract. 2017; 26(6):547-53.

3. Chung S, Youn S, Park B, Lee S, Kim C. A sleep education and hypnotics reduction program for hospitalized patients at a general hospital. Psychiatry Investig. 2018;15(1):78-83.

4. Abad VC, Guilleminault C. Insomnia in elderly patients: recommendations for pharmacological management. Drugs Aging. 2018;35(9):791-817.

5. Mendelson WB. A review of the evidence for the efficacy and safety of trazodone in insomnia. J Clin Psychiatry. 2005;66(4):469-76.

6. Frels C, Williams P, Narayanan S, Gariballa SE. Iatrogenic causes of falls in hospitalised elderly patients: a case-control study. Postgrad Med J. 2002; 78(922):487-9.

7. Kolla BP, Lovely JK, Mansukhani MP, Morgenthaler TI. Zolpidem is independently associated with increased risk of inpatient falls. J Hosp Med. 2013;8(1):1-6.

8. Pavon JM, Zhao Y, McConnell E, Hastings SN. Identifying risk of readmission in hospitalized elderly adults through inpatient medication exposure. J Am Geriatr Soc. 2014;62(6):1116-21.

9. Tamiya H, Yasunaga H, Matusi H, Fushimi K, Ogawa S, Akishita M. Hypnotics and the occurrence of bone fractures in hospitalized dementia patients: a matched case-control study using a national inpatient database. PLoS One. 2015;10(6):e0129366.

10. Bloch F, Thibaud M, Dugue B, Breque C, Rigaud AS, Kemoun G. Psychotropic drugs and falls in the elderly people: updated literature review and meta-analysis. J Aging Health. 2011;23(2):329-46.

11. Cashin RP, Yang M. Medications prescribed and occurrence of falls in general medicine inpatients. Can J Hosp Pharm. 2011;64(5):321-6.

12. Macri JC, Iaboni A, Kirkham JG, Maxwell C, Gill SS, Vasudev A, et al. Association between antidepressants and fall-related injuries among longterm care residents. Am J Geriatr Psychiatry. 2017;25(12):1326-36.

13. Coupland C, Dhiman P, Morriss R, Arthur A, Barton G, Hippisley-Cox J. Antidepressant use and risk of adverse outcomes in older people: population based cohort study. BMJ. 2011;343:d4551.

14. Iaboni A, Bronskill SE, Reynolds KB, Wang X, Rochon PA, Herrmann N, et al. Changing pattern of sedative use in older adults: a population-based cohort study. Drugs Aging. 2016;33(7):523-33.

15. Schumacher L, Dobrinas M, Tagan D, Sautebin A, Blanc AL, Widmer N. Prescription of sedative drugs during hospital stay: a Swiss prospective study. Drugs Real World Outcomes. 2017;4(4):225-34.

16. Gillis CM, Poyant JO, Degrado JR, Ye L, Anger KE, Owens RL. Inpatient pharmacological sleep aid utilization is common at a tertiary medical center. J Hosp Med. 2014;9(10):652-7.

17. Bell CM, Fischer HD, Gill SS, Zagorski B, Sykora K, Wodchis WP, et al. Initiation of benzodiazepines in the elderly after hospitalization. J Gen Intern Med. 2007;22(7):1024-9.

18. Grad R, Tamblyn R, Holbrook AM, Hurley J, Feightner J, Gayton D. Risk of a new benzodiazepine prescription in relation to recent hospitalization. J Am Geriatr Soc. 1999;47(2):184-8.
19. Reeve E, Ong M, Wu A, Jansen J, Petrovic M, Gnjidic D. A systematic review of interventions to deprescribe benzodiazepines and other hypnotics among older people. Eur J Clin Pharmacol. 2017;73(8):927-35.

20. 2019 American Geriatrics Society Beers Criteria ${ }^{\circledR}$ Update Expert Panel. American Geriatrics Society 2019 updated AGS Beers criteria ${ }^{\circledR}$ for potentially inappropriate medication use in older adults. J Am Geriatr Soc. 2019;67(4):674-94.

21. O'Mahony D, O'Sullivan D, Byrne S, O'Connor MN, Ryan C, Gallagher P. STOPP/START criteria for potentially inappropriate prescribing in older people: version 2. Age Ageing. 2015;44(2):213-8.

22. Alessi-Severini S, Bolton JM, Enns MW, Dahl M, Collins DM, Chateau D, et al. Use of benzodiazepines and related drugs in Manitoba: a populationbased study. CMAJ Open. 2014;2(4):E208-16.

23. Huerta C, Abbing-Karahagopian V, Requena G, Oliva B, Alvarez Y, Gardarsdottir $\mathrm{H}$, et al. Exposure to benzodiazepines (anxiolytics, hypnotics and related drugs) in seven European electronic healthcare databases: a cross-national descriptive study from the PROTECT-EU project. Pharmacoepidemiol Drug Saf. 2016;25 Suppl 1:56-65.

24. Weymann D, Gladstone EJ, Smolina K, Morgan SG. Long-term sedative use among community-dwelling adults: a population-based analysis. CMAJ Open. 2017;5(1):E52-60.

25. Pan-Canadian trends in the prescribing of opioids and benzodiazepines, 2012 to 2017. Canadian Institute for Health Information; 2018.

26. Cadogan CA, Ryan C, Cahir C, Bradley CP, Bennett K. Benzodiazepine and Z-drug prescribing in Ireland: analysis of national prescribing trends from 2005 to 2015. Br J Clin Pharmacol. 2018;84(6):1354-63.

27. Maust DT, Blow FC, Wiechers IR, Kales HC, Marcus SC. National trends in antidepressant, benzodiazepine, and other sedative-hypnotic treatment of older adults in psychiatric and primary care. J Clin Psychiatry. 2017;78 (4):e363-71.

28. Brunero $S$, Wand AP, Lamont S, John L. A point prevalence study of the use of psychotropic medication in an acute general hospital. Int Psychogeriatr. 2016;28(6):967-75.

29. Pek EA, Remfry A, Pendrith C, Fan-Lun C, Bhatia RS, Soong C. High prevalence of inappropriate benzodiazepine and sedative hypnotic prescriptions among hospitalized older adults. J Hosp Med. 2017;12(5):310-6.

30. Arnold I, Straube K, Himmel W, Heinemann S, Weiss V, Heyden L, et al. High prevalence of prescription of psychotropic drugs for older patients in a general hospital. BMC Pharmacol Toxicol. 2017;18(1):Article 76.

31. Ng BJ, Le Couteur DG, Hilmer SN. Deprescribing benzodiazepines in older patients: impact of interventions targeting physicians, pharmacists, and patients. Drugs Aging. 2018;35(6):493-521.

32. Ansari F, Erntell M, Goossens H, Davey P. The European surveillance of antimicrobial consumption (ESAC) point-prevalence survey of antibacterial use in 20 European hospitals in 2006. Clin Infect Dis. 2009;49(10): 1496-504.

33. Zarb P, Amadeo B, Muller A, Drapier N, Vankerckhoven V, Davey P, et al. Identification of targets for quality improvement in antimicrobial prescribing: the web-based ESAC point prevalence survey 2009.J Antimicrob Chemother. 2011;66(2):443-9.

34. Table 17-10-0005-0: Population estimates on July 1st, by age and sex. Statistics Canada; [cited 2019 Dec 4]. Available from: https://doi.org/ 10.25318/1710000501-eng

35. Canada's health care system. Government of Canada; 2016 [cited 2019 Dec 4]. Available from: https://www.canada.ca/en/health-canada/services/canadahealth-care-system.html

36. About us. Nova Scotia Health Authority; 2020 [cited 2020 May 28]. Available from: www.nshealth.ca/about-us

37. Nova Scotia Health Authority: by the numbers 2015-16. In: Annual report 2015-16. Nova Scotia Health Authority; 2016 [cited 2020 May 28]. Available from: www.nshealth.ca/AnnualReport2016/numbers.html

38. Alternate level of care in Canada. Canadian Institute for Health Information; 2009 Jan 14.

39. Population centre (POPCTR). In: Dictionary, census of population, 2016. Statistics Canada; 2019 [cited 2019 Dec 4]. Available from: https://www12. statcan.gc.ca/census-recensement/2016/ref/dict/geo049a-eng.cfm 
40. Elliott RA, Woodward MC, Oborne CA. Improving benzodiazepine prescribing for elderly hospital inpatients using audit and multidisciplinary feedback. Intern Med J. 2001;31(9):529-35.

41. Khawaja MR, Majeed A, Malik F, Merchant KA, Maqsood M, Malik R, et al. Prescription pattern of benzodiazepines for inpatients at a tertiary care university hospital in Pakistan. J Pak Med Assoc. 2005;55(6):259-63.

42. Lavan AH, Gallagher P. Predicting risk of adverse drug reactions in older adults. Ther Adv Drug Saf. 2016;7(1):11-22.

43. Soong C, Leis J. Less sedatives for your older relatives: a toolkit for reducing inappropriate use of benzodiazepines and sedative-hypnotics among older adults in hospital. Choosing Wisely Canada; 2017 [cited 2018 Oct 5]. Available from: https://choosingwiselycanada.org/wp-content/uploads/2017/07/ CWC_BSH_Hospital_Toolkit_v1.3_2017-07-12.pdf

44. Zopiclone. In: Compendium of pharmaceuticals and specialties. Canadian Pharmacists Association; 2019 [cited 2019 Dec 4]. Available from: www. e-cps.ca. Subscription required to access content.

45. Halme AS, Beland SG, Preville M, Tannenbaum C. Uncovering the source of new benzodiazepine prescriptions in community-dwelling older adults'. Int J Geriatr Psychiatry. 2013;28(3):248-55.

46. Freter S, Koller K, Dunbar M, MacKnight C, Rockwood K. Translating delirium prevention strategies for elderly adults with hip fracture into routine clinical care: a pragmatic clinical trial. JAm Geriatr Soc. 2017;65(3):567-73.

47. Wilson MG, Lee TC, Hass A, Tannenbaum C, McDonald EG. EMPOWERing hospitalized older adults to deprescribe sedative hypnotics: a pilot study. J Am Geriatr Soc. 2018;66(6):1186-9.

48. Mitchell MD, Gehrman P, Perlis M, Umscheid CA. Comparative effectiveness of cognitive behavioral therapy for insomnia: a systematic review. BMC Fam Pract. 2012;13:Article 40.

Heather L Neville, BSCPharm, MSc, FCSHP, is with the Nova Scotia Health Authority, Halifax, Nova Scotia.

Mia Losier, BScPharm, ACPR, was, at the time of this study, with the Nova Scotia Health Authority and the College of Pharmacy, Dalhousie University, Halifax, Nova Scotia. She is now with Horizon Health Network, Saint John, New Brunswick.
Jennifer Pitman, BSCMedSC, BScPharm, ACPR, was, at the time of this study, with the Nova Scotia Health Authority and the College of Pharmacy, Dalhousie University, Halifax, Nova Scotia. She is now with the Vancouver Island Health Authority, Victoria, British Columbia.

Melissa Gehrig, BSc(Hons), BScPharm, MSc, is with the Nova Scotia Health Authority, Halifax, Nova Scotia.

Jennifer E Isenor, BScPharm, PharmD, is with the College of Pharmacy, Dalhousie University, Halifax, Nova Scotia.

Laura V Minard, BSc, BScPharm, ACPR, PhD, is with the Nova Scotia Health Authority, Halifax, Nova Scotia.

Ellen Penny, BScPharm, PharmD, BCGP, is with the Nova Scotia Health Authority, Sydney, Nova Scotia.

Susan K Bowles, BScPhm, MSc, PharmD, FCSHP, is with the Nova Scotia Health Authority and the College of Pharmacy, Dalhousie University, Halifax, Nova Scotia.

Competing interests: Jennifer Isenor has received grants from Sanofi and GlaxoSmithKline for research unrelated to the work described here. No other competing interests were declared.

\section{Address correspondence to:}

Heather L Neville

Victoria General Hospital, Nova Scotia Health Authority

Room 2043, 1276 South Park Street

Halifax NS B3H 2 Y9

e-mail: Heather.neville@nshealth.ca

Funding: This study was financially supported by the Drug Evaluation Alliance of Nova Scotia (DEANS) and the office of the Vice-President of Research of the Nova Scotia Health Authority.

Disclaimer: Susan Bowles is an Associate Editor with the Canadian Journal of Hospital Pharmacy. She was not involved in the editorial decision-making process for this article. 